\title{
Physical properties and dynamical relation of the circular depressions on comet 67P/Churyumov-Gerasimenko
}

\author{
W.-H. Ip ${ }^{1,2,3}$, I.-L. Lai ${ }^{2}$, J.-C. Lee ${ }^{4}$, Y.-C. Cheng ${ }^{1}$, Y. Li ${ }^{3}$, Z.-Y. Lin ${ }^{1}$, J.-B. Vincent ${ }^{5}$, S. Besse ${ }^{6}$, H. Sierks ${ }^{5}$, \\ C. Barbieri ${ }^{7}, 8$, P. L. Lamy ${ }^{9}$, R. Rodrigo ${ }^{10,11}$, D. Koschny ${ }^{6}$, H. Rickman ${ }^{12,13}$, H. U. Keller ${ }^{14}$, J. Agarwal ${ }^{5}$, \\ M. F. A'Hearn ${ }^{15}$, M. A. Barucci ${ }^{16}$, J.-L. Bertaux ${ }^{17}$, I. Bertini ${ }^{8}$, D. Bodewits ${ }^{15}$, S. Boudreault ${ }^{5}$, G. Cremonese ${ }^{18}$, \\ V. Da Deppo ${ }^{19}$, B. Davidsson ${ }^{12}$, S. Debei ${ }^{20}$, M. De Cecco ${ }^{21}$, M. R. El-Maarry ${ }^{22}$, S. Fornasier ${ }^{16}$, M. Fulle ${ }^{23}$, \\ O. Groussin ${ }^{9}$, P. J. Gutiérrez ${ }^{24}$, C. Güttler ${ }^{5}$, S. F. Hviid ${ }^{25}$, L. Jorda ${ }^{9}$, J. Knollenberg' ${ }^{25}$, G. Kovacs ${ }^{5}$, J.-R. Kramm ${ }^{5}$, \\ E. Kührt ${ }^{25}$, M. Küppers ${ }^{26}$, F. La Forgia ${ }^{7}$, L. M. Lara ${ }^{24}$, M. Lazzarin ${ }^{7}$, J. J. López-Moreno ${ }^{24}$, S. Lowry ${ }^{27}$, \\ S. Marchi ${ }^{28}$, F. Marzari ${ }^{7}$, H. Michalik ${ }^{14}$, S. Mottola ${ }^{25}$, G. Naletto ${ }^{8,19,29}$, N. Oklay ${ }^{5}$, M. Pajola ${ }^{8}$, N. Thomas ${ }^{22}$, \\ E. $\operatorname{Toth}^{30}$, and C. Tubiana ${ }^{5}$ \\ (Affiliations can be found after the references)
}

Received 19 January 2016 / Accepted 18 April 2016

\begin{abstract}
Aims. We aim to characterize the circular depressions of comet 67P/Churyumov-Gerasimenko and investigate whether such surface morphology of a comet nucleus is related to the cumulative sublimation effect since becoming a Jupiter family comet (JFC).

Methods. The images from the Rosetta/OSIRIS science camera experiment are used to construct size frequency distributions of the circular depression structures on comet 67P and they are compared with those of the JFCs 81P/Wild 2, 9P/Tempel 1, and 103P/Hartley 2. The orbital evolutionary histories of these comets over the past $100000 \mathrm{yr}$ are analyzed statistically and compared with each other.

Results. The global distribution of the circular depressions over the surface of 67P is charted and classified. Descriptions are given to the characteristics and cumulative size frequency distribution of the identified features. Orbital statistics of the JFCs visited by spacecraft are derived.

Conclusions. The size frequency distribution of the circular depressions is found to have a similar power law distribution to those of 9P/Tempel 1 and $81 \mathrm{P} /$ Wild 2 . This might imply that they could have been generated by the same process. Orbital integration calculation shows that the surface erosion histories of 81P/Wild 2, and 9P/Tempel 1 could be shorter than those of 67P, 103 P/Hartley 2 and 19P/Borrelly. From this point of view, the circular depressions could be dated back to the pre-JFC phase or the transneptunian phase of these comets. The north-south asymmetry in the distribution of the circular depressions could be associated with the heterogeneous structure of the nucleus of comet 67P and/or the solar insolation history.
\end{abstract}

Key words. comets: individual: 67P/Churyumov-Gerasimenko

\section{Introduction}

As the most primitive objects of icy composition from the outer solar system, the chemical composition, surface morphology and internal structure of comets carry key information on the formation of the solar system. Because of the high volatility of the condensed water ices, active comets in general exhibit visible outgassing phenomena when they move inside the socalled snow line at a heliocentric distance of about 2.5 au. Depending on the orbital configuration, the evolutionary history and the rotational state, the surface structures and size of a comet nucleus could be changed considerably as a consequence of global outgassing processes and localized mass wasting mechanisms (Basilevsky \& Keller 2006; Belton 2010; Keller et al. 2015). In the first report of the OSIRIS science imaging results, Sierks et al. (2015) suggested that the nucleus of 67P/Churyumov-Gerasimenko (67P hereafter) could be just one single monolith (see also Thomas et al. 2015). Subsequently, Massironi et al. (2015), showed that a contact binary model with the two lobes being completely different entities or cometesimals could be an explanation (see also Rickman et al. 2015; Davidsson et al. 2016; El-Maarry et al. 2015a). Similar scenarios may be proposed for the dumb-bell shaped structures of comet 103P/Hartley 2, comet 1P/Halley and comet 19P/Borrelly even though the shapes of comet 9P/Tempel 1 and 81P/Wild 2 are certainly more monolithic. Spacecraft close-up observations reveal that these bodies have something in common concerning the presence on the surface of many pits or quasi-circular flat-floor depression structures (circular depressions or pits hereafter; see Fig. 1). These structures are different from the bowlshape structure of the impact craters found on the Moon and asteroids. These circular depressions are characterized by steep walls and flat bottoms similar to those first found by the Stardust mission at comet 81P/Wild 2 (Brownlee et al. 2004). Subsequent observations at comet 9P/Tempel 1 by the Deep Impact spacecraft showed the presence of similar circular depressions in some regions (Belton et al. 2013) with comet 103P/Hartley 2 sharing similar features (Bruck Syal et al. 2013).

According to Belton et al. (2013), there were 380 circular depressions with diameters of between $25 \mathrm{~m}$ and $940 \mathrm{~m}$ on the surface of comet 9P/Tempel 1 . They were concentrated mostly in the equatorial region where several outbursts were detected in some localized areas. From a study of the cumulative size frequency distributions of these structures on comet 9P/Tempel 1 and 81P/Wild 2, Belton et al. (2013) found that they could be fitted by a power law $N(D)$ with the same power law index of $k=2.24$ where $N(D)$ is the number of circular depressions with 

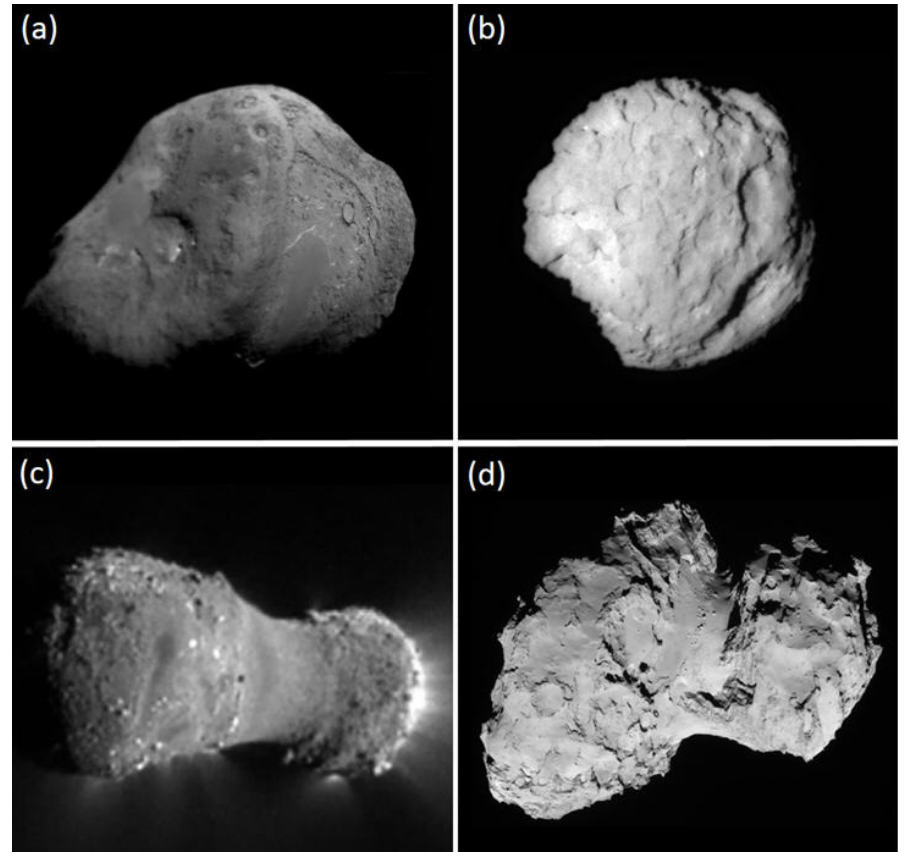

Fig. 1. A family portrait of the comets imaged by recent space missions a) comet $9 \mathrm{P} /$ Tempel 1 ; b) comet $81 \mathrm{P} /$ Wild 2 ; c) comet $103 \mathrm{P} /$ Hartley 2 and d) comet $67 \mathrm{P}$ Churyumov-Gerasimenko. Some circular depressions are discernable in these images. Image credits: a)-c) NASA; d) ESA/MPS.

diameters larger than $D$. One way to examine whether the circular depressions on comet 67P and these two comets are related by similar process is thus to compare their size frequency distributions.

Since meteoroid impact is considered to be too infrequent to produce craters on the nucleus surface of $67 \mathrm{P}$ pockmarked by circular depressions, some other mechanisms such as sinkhole collapse (Vincent et al. 2015a) or explosive outbursts (Belton et al. 2013) have been proposed. It is important, therefore, to ask whether sudden mass-wasting events or more gradual outgassing processes are associated with the formation of the circular depressions. Indeed, observations by the OSIRIS science camera showed increasing level of coma activity, dust jet formation and terrain changes during approach to perihelion $(q)$ at 1.2 au (Lara et al. 2015; Lin et al. 2015, 2016; Vincent et al. 2015a; Groussin et al. 2015). This means that the global morphologies of cometary nucleus surfaces could be somehow determined by the "sublimation erosion age", namely, how much time in total the comets have spent inside the snow line. To examine this plausible connection, we need to consider the past orbital histories of these comets. The main thing is to estimate the cumulative time a comet has been subject to active outgassing in the recent past since becoming a Jupiter family comet (JFC).

In brief, the paper is structured as follows. Section 2 will describe the physical properties and distribution of the circular depressions on comet 67P with a view to comparing them to similar features on the other comets with close-up imaging observations by spacecraft. The results of orbital integration simulating the long-term evolution of the orbits of comets subject to the gravitational perturbations of the giant planets are presented in Sect. 3. A summary and discussion of the two different lines of investigation and the ordering in terms of the sublimation age are presented in Sect. 4.

\section{Observations}

Figure 2 shows three types of circular depressions found on $67 \mathrm{P}$. The first is the typical steep-wall and flat bottom structure (see Fig. 2a). A number of boulders can be identified on the floor of the depression. The second kind is characterized by the presence of large gaps, and thus is given the name "half-pit". This shape might be due to partial collapse via slope movement in one preferred direction (see Fig. 2b). Such a scenario of slope retreat has been discussed in the context of comets and sublimation erosion by Britt et al. (2004). Finally, as discovered by Vincent et al. (2015a), several circular features showed jet activity (see Fig. 2c).

Figures 3 and 4 show the locations of the circular depression features on different sides of the nucleus surface. The center coordinates of these structures are summarized in Table A.1. In total, 56 can be identified, most of which are located in Seth and Ash on one side of Hapi (Figs. 3a-c and 4c), Bastet and Ma' at in the Head (Figs. 3d and 4b). They are not present in the smooth terrains of Hapi. Only a few circular depressions exist on the southern part of the nucleus. The active pit, Seth 01, described in Vincent et al. (2015a) has also been included. It is interesting to note that Seth 01 , which has a diameter of $220 \mathrm{~m}$, is smaller than many of the circular depressions that did not show material outflows. This is probably because the observed activity investigated by Vincent et al. (2015a) when 67P was moving from 3.80 au to 2.65 au could have been driven by the sublimation of ices such as $\mathrm{CO}$ and $\mathrm{CO}_{2}$ that are more volatile than water ice. This might also mean that the larger flat-floored pits could be older than Seth 01.

Figure 5 is a 2D map of the distribution of circular depressions on a Mercator projection. Two striking features can be seen. Firstly, there are very few pits in the southern hemisphere which was sunlit only for a short time near and after perihelion. Secondly, some of the circular depressions in the northern hemisphere appear to have a tendency to align along a cliff-wall like structure (A in Fig. 5). Another set seems to line up around the rim of the Hatmehit basin (B in Fig. 5). Following the outlines of $\mathrm{A}$ and $\mathrm{B}$, some circular depressions would be expected on the southern hemisphere. But very few can be found.

Previous studies of hypervelocity impacts on fluffy materials have shown that the resultant craters generally have bowlshaped bottoms (Housen \& Holsapple 2003; Schultz et al. 2007; Yasui et al. 2012). This is one reason for believing that the circular depressions found on 67P and other comets with steep-walls and flat-bottoms are not of impact origin although we cannot rule out that some of the pits may have been related to impact events. A fracture developed by thermal stress or tectonic stress, or a crater generated by the explosive loss of volatiles could all be the possible source mechanism. An alternative scenario therefore, is to suggest that these geological structures were first initiated by point impacts by interplanetary meteoroids to be followed by lateral expansion of the circumference without increasing the depth significantly (Cheng et al. 2013). This argument is weakened by the Rosetta finding that the southern hemisphere is relatively free of circular depressions.

Indeed, for a recent meteoroid bombardment origin, the existence of some circular pits would be expected in the Hapi region of 67P, and some smooth areas on 9P/Tempel 1 (Belton et al. 2013), and the neck region of 103P/Hartley 2 (Bruck Syal et al. 2013). In any event, it has been pointed out by Belton \& Melosh (2009) and subsequently Vincent et al. (2015b) that impact process in the main asteroid belt is not sufficient to account for the 


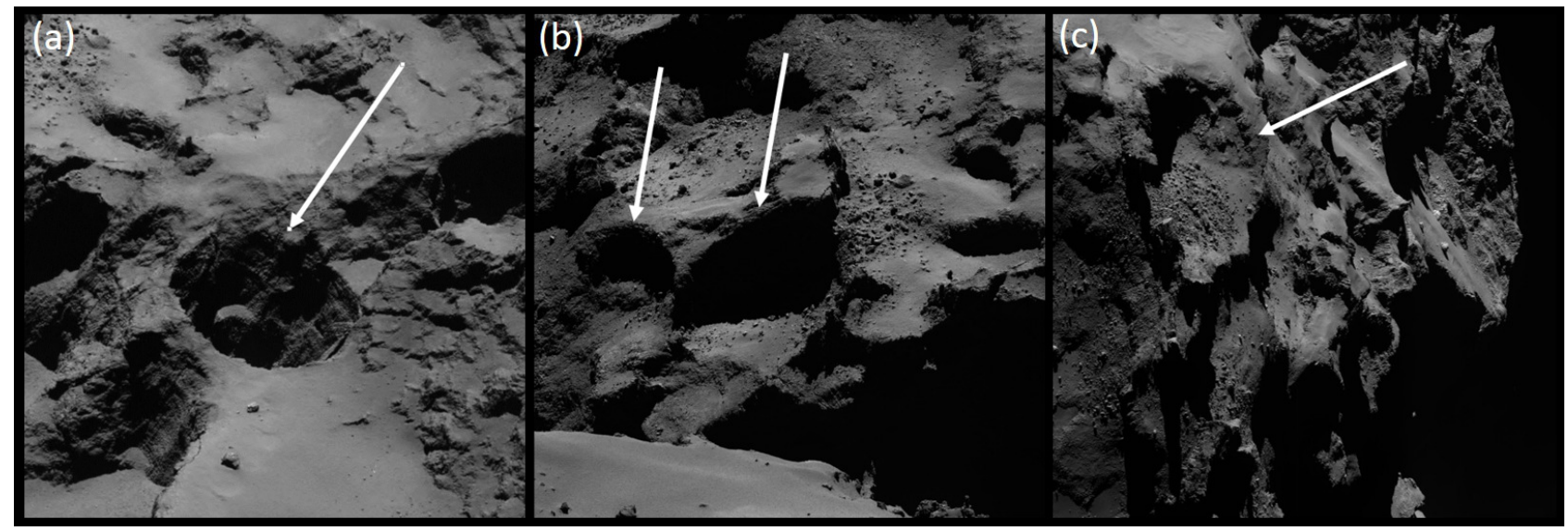

Fig. 2. Close-up views of three circular depressions: a) the active pit S19 (Seth-01 in Vincent et al. 2015a)-NAC-2014-09-22T02.34.12.847Z-ID301397549000-F16; b) two half-pits in Seth, S12 and S13-NAC-2014-10-01T23.56.06.564Z-ID30-1397549900-F22; c) a regular circular depression in Ash, A17-NAC-2014-09-19T15.09.52.414Z-ID30-1397549001-F41.

number of the pits. The impact history prior to crossing of the water snow line will be discussed later.

Figure 6 shows the differential number distribution of the circular depressions as a function of the $d / D$ ratio. The statistics performed are as follows. We first identify the possible features by comparing their morphological structures and the depth-todiameter $(d / D)$ ratios. In order to make quantitative assessment of the distribution of the circular depressions of different sizes, we have used the digital data of the shape model (Preusker et al. 2015) instead of the OSIRIS images. It can be seen that most of them have $d / D \leq 0.2$ while a small fraction have $d / D>0.3$. The half-pits tend to have large depth values (i.e., $d / D>0.4$ ). If we examine the trilateral relationship between size, $d / D$ and activity, we will find the interesting situation that the active circular depressions (or pits) examined by Vincent et al. (2015a) are all small $(<400 \mathrm{~m}$ ), with an intermediate $d / D$ ratio of about 0.4 (see Fig. 7).

Figure 8 shows the cumulative size frequency distribution of the circular depressions on comet 67P for $D>150 \mathrm{~m}$, obtained from the technique described in Lamy et al. (2004). If all data points are included when fitting to a power law distribution in the form of $N(D) \sim D^{-k}$, we obtain $k=1.8 \pm 0.3$. But if only circular depressions with $D>200 \mathrm{~m}$ are considered, as in the previous work of Belton et al. (2013), $k=2.3 \pm 0.2$. It is interesting that this $k$ value of $67 \mathrm{P}$ is very similar to the value of 2.24 determined for comets $9 \mathrm{P}$ and $81 \mathrm{P}$ by these authors. While the physical process responsible for such power law distribution is not yet known, the similarity of the $k$ values of these three comets is consistent with a common origin.

\section{Comparison of orbital histories}

From orbital integration backward in time, it has been shown that comet 67P's perihelion was lowered from 2.7 au (outside the snow line) to 1.3 au via a close encounter with Jupiter in 1959 and subsequently reduced to 1.2 au (Groussin et al. 2007; Maquet 2015). There is therefore the general consensus that 67P could represent a fresh-faced comet nucleus - with a very geologically young surface that is out of equilibrium with the thermal regime. Similar orbital calculations have also indicated that the perihelion of comet $81 \mathrm{P} /$ Wild 2 was changed from 5 au to $1.5 \mathrm{au}$ in 1974 (Sekanina 2003). It should also be young. This might be the case; however, cometary orbits can vary widely because of planetary scattering in their dynamical histories since injection from their source regions such as the Kuiper belt. Many comets could move back and forth between the short-period and long-period population during their dynamical evolution (Levison \& Duncan 1994; Rickman et al. 2015). To estimate the integral outgassing effect, we must examine their corresponding orbital behaviors before the most recent perihelion-lowering event.

We have performed backward orbital integration using the Mercury package (Chambers 1999) for the five comets: 9P/ Tempel 1, 19P/Borrelly, 67P, 81P/Wild 2, and 103P/Hartley 2 . In the numerical calculations, all four giant planets are included with the masses of the terrestrial planets being added to the Sun. The time step used is one day. Figure 9 shows the time variations of perihelion $(q)$, of over the last $1000 \mathrm{yr}$ without taking the nongravitational force terms into account. The abrupt changes in $q$ were caused by close encounters with Jupiter.

We find that comet $81 \mathrm{P} /$ Wild 2 spent most of its time outside the snow line at 2.5 au except for over the last $40 \mathrm{yr}$ since 1974 (Sekanina 2003). A different history for the orbit of 67P is found if the parameters given in JPL Horizons ephemeris system for 67P in epoch 2015 are used instead of those given in 1969. The older version (in blue dashed line) indicates that 67P should have its $q$ value $>2.5$ au before 1959 . The newer version would require $67 \mathrm{P}$ to stay inside or near the snow line for a good fraction of the past one thousand years. In comparison, 9P/Tempel 2 reached outside the snow line intermittently until $500 \mathrm{yr}$ ago. On the other hand, the perihelia of 19P/Borrelly and 103P/Hartley 2 always have $q$ values smaller than 2.5 au. These short-term calculations imply that the surface of $81 \mathrm{P}$ should be very young and those of 19P, and 103P very old from the point of view of erosive sublimation process. But what about their long-term orbital evolutions? Could 81P/Wild 2 have moved to orbits with $q<2.5 \mathrm{au}$ at an earlier time such that its surface might have been subject to stronger aging effects? Alternatively, 9P and 67P could have wandered into low- $q$ orbits only a thousand years ago. The same might hold true for 19P and 103P. According to Levison \& Duncan (1994) a typical JFC has a dynamical lifetime of $0.33 \mathrm{Myr}$, of which $7 \%$ of the time is spent on orbits with $q<2.5$ au (hence "visible") with potential water sublimation. Typically a JFC will switch between being a "visible" comet, and being an icy object with much larger perihelion distance about ten times.

Another important factor is that in such dynamical simulations close encounters with the giant planets would introduce 


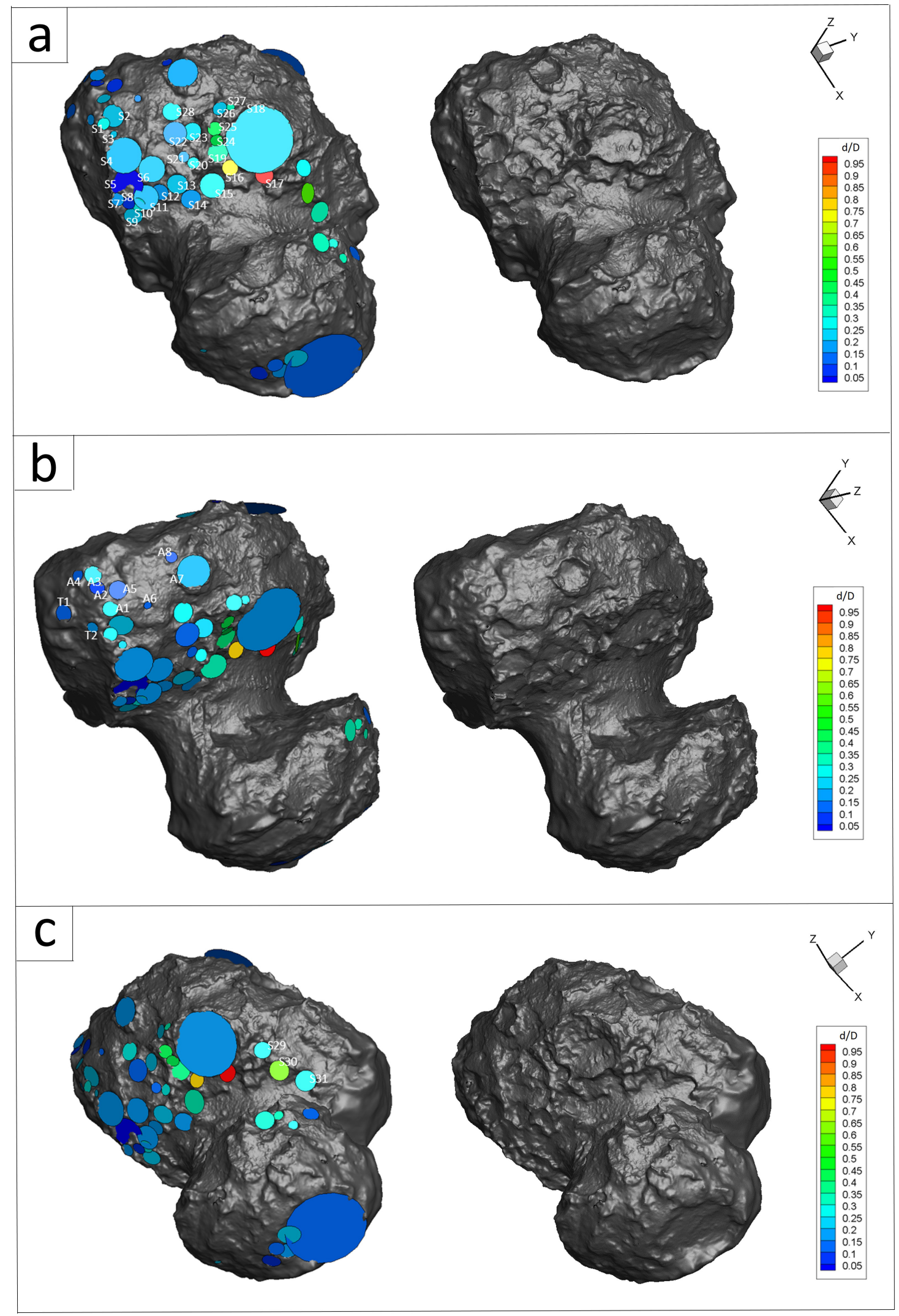

Fig. 3. Identification of the circular depressions on different sides of the comet nucleus surface: a) Seth; b) Ash and Atum; and c) Seth. The picture on the right-hand side of each panel is shown for comparison with the markings of the circular depression structures on the left-hand side. The depth-to-diameter ratios $(d / D)$ are determined by using the digital data of the shape model.

numerical uncertainties into the orbital integration making the trajectory computation less and less exact. This implies that with longer the numerical calculations there will be a higher level of statistical fluctuation as a result of the cumulative effect of the non-gravitational force and random planetary gravitational scattering. For example, Groussin et al. (2015) discussed the 


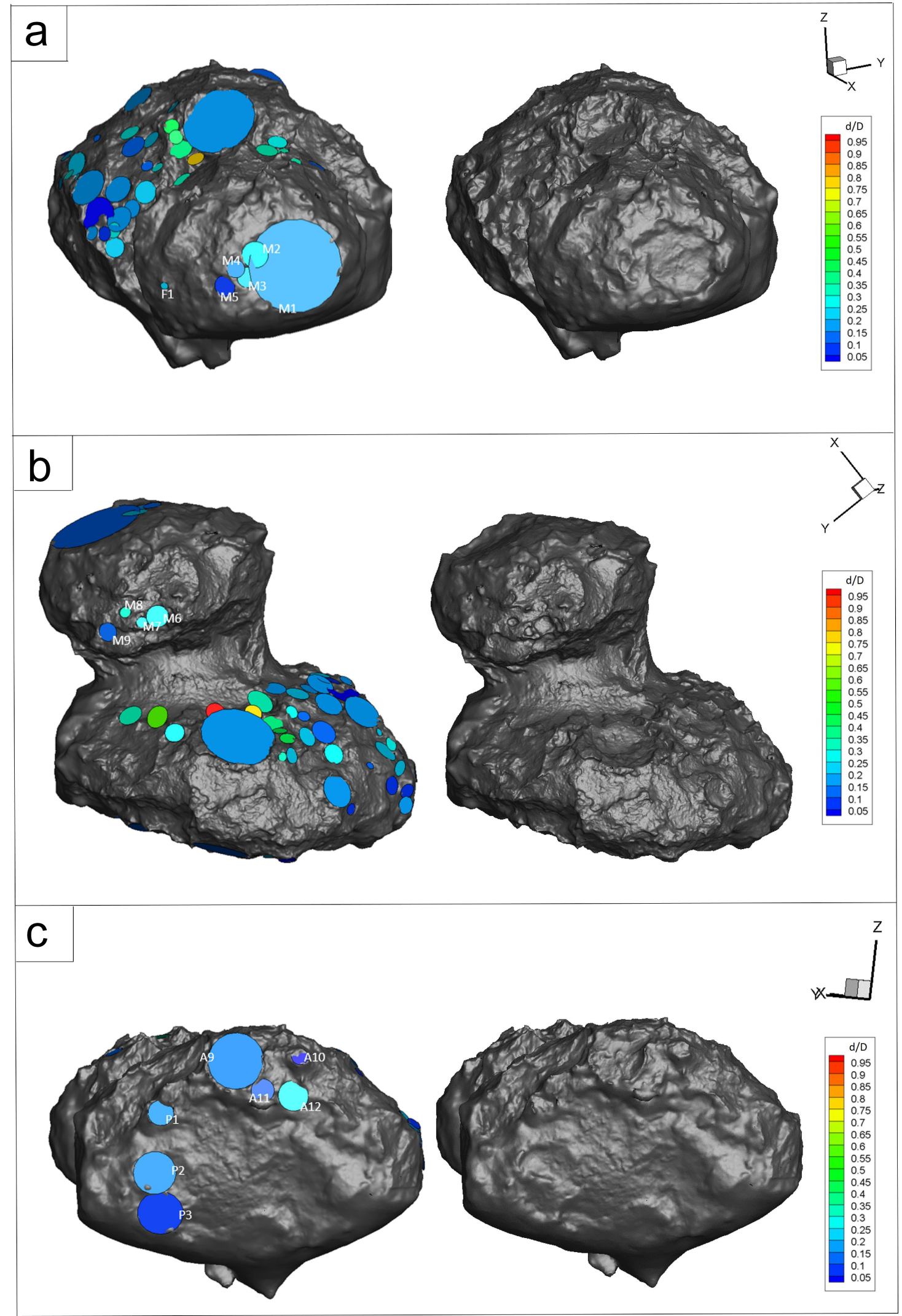

Fig. 4. Same as Fig. 3 for a) Hatmehit, Ma'at, and Maftet; b) Ma'at; and c) Ash and Imhotep.

chaotic orbital dynamics of both 46P/Wirtanen and 67P coupled to the mass loss process; a wide range of changes in perihelion distances can be attained by model calculations with or without non-gravitational forces. In order to evaluate the general trend of the evolutionary histories of individual comets, we follow the statistical method of tracing the orbital evolution of each of them by generating a number of clones. The orbital elements of these clone particles are created based on the covariance matrix 


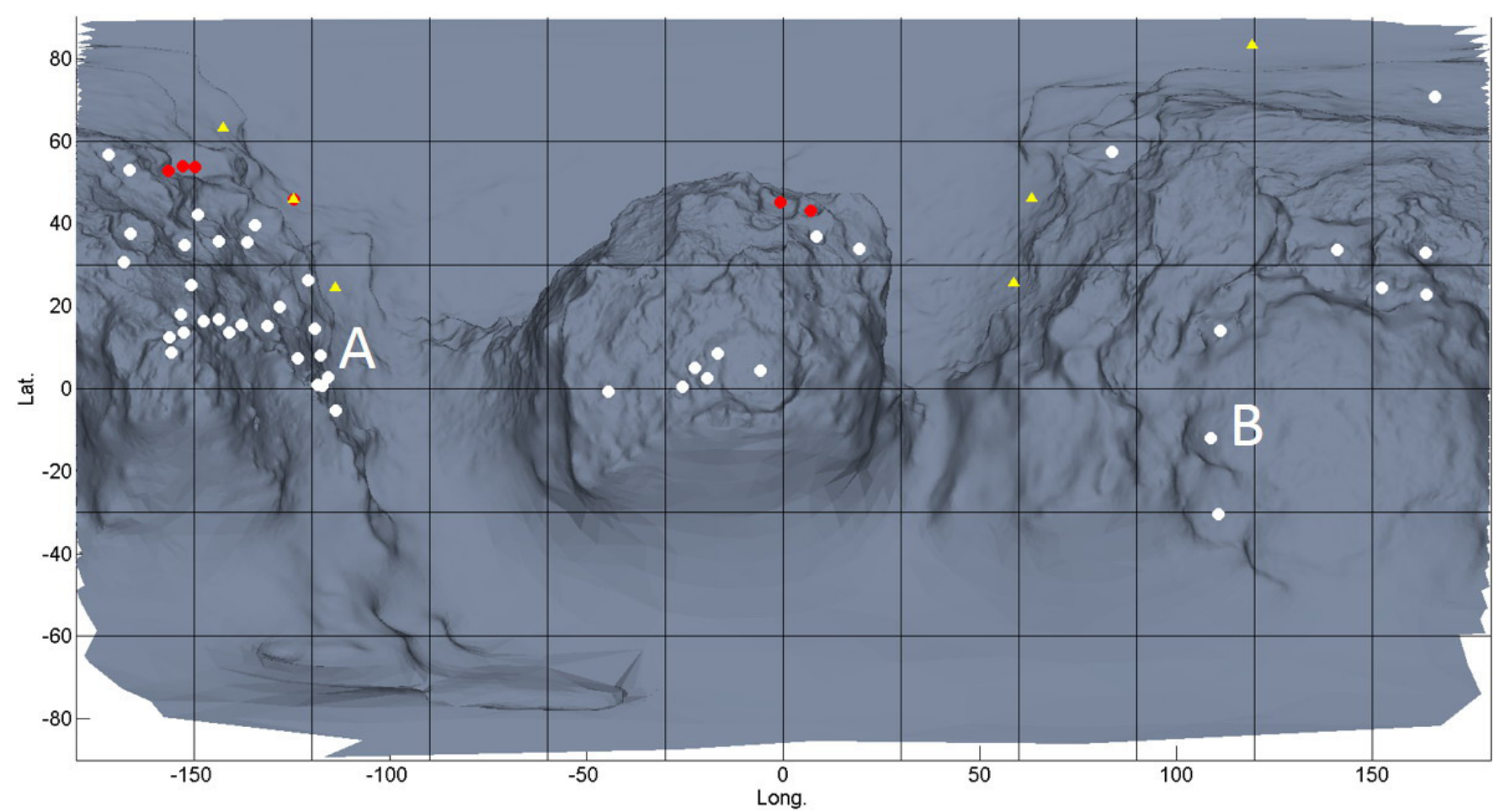

Fig. 5. Distribution of the circular depressions on a Mercator projection map. The circles filled in red are the active pits in Vincent et al. (2015a). The triangles filled in yellow are the half pits near Hapi.

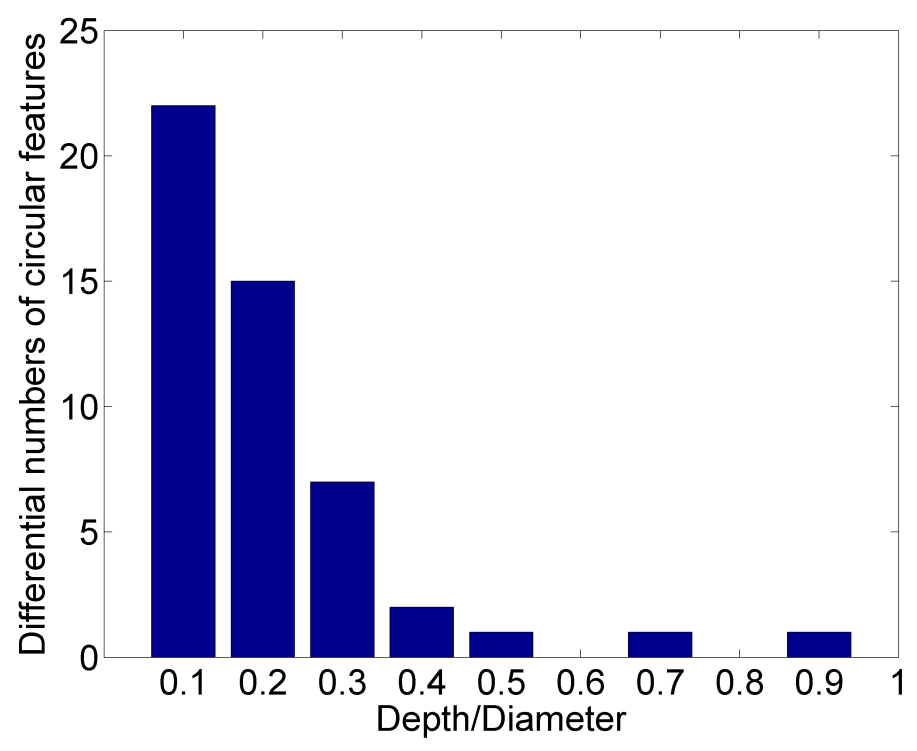

Fig. 6. The differential number distribution of the circular depressions as a function of the depth-to-Diameter $(d / D)$ ratio.

provided by JPL Small-Body Data Base which are in a Gaussian distribution within one-sigma uncertainty for each of the given orbital elements. The total integration time is $100000 \mathrm{yr}$ for each case because the average physical lifetime of shortperiod comets limited by either the buildup of a dust mantle or explosive disruption - has been derived to be only several thousand years by a number of studies (Fernández et al. 2002, 2014; Brasser \& Wang 2015).

Figure 10 compares the results of the backward orbital integrations of the 1000 clones for each of the five comets. The

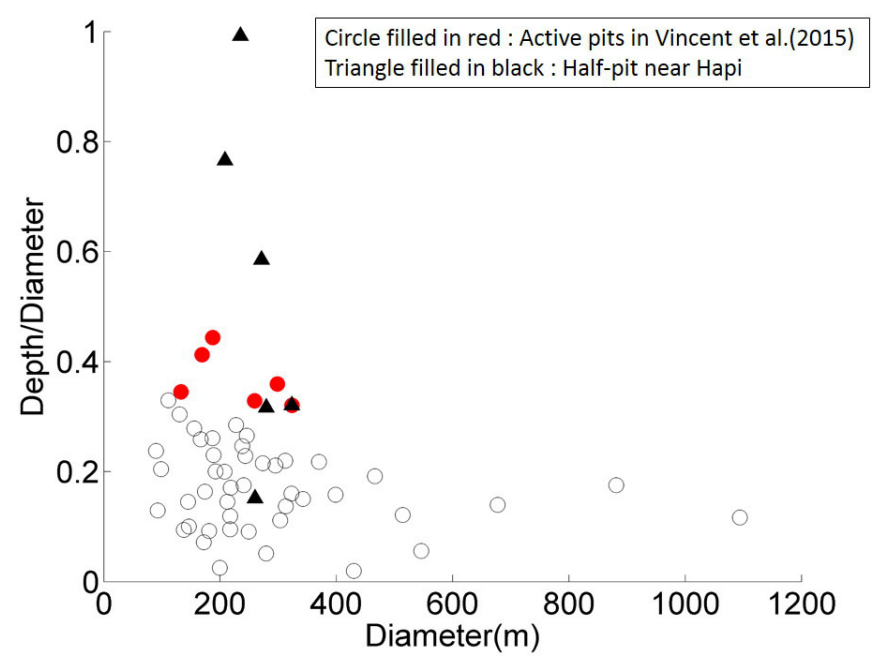

Fig. 7. Circular depressions plotted by size (diameter) and depth-todiameter $(d / D)$ ratio. The active circular depressions (pits) found by Vincent et al. (2015a) are marked in filled red circles and the half pits in filled black triangles.

ordering is based on the following analysis of the surface erosion histories :

(a) The nucleus surface of $81 \mathrm{P} /$ Wild 2 should be young since most of the clone orbits do not have perihelia smaller than the snow line at 2.5 au for the last $100000 \mathrm{yr}$.

(b) $9 \mathrm{P} /$ Tempel 1 has a small probability of possessing $q<2.5$ au in the past $100000 \mathrm{yr}$. It is possible that it experienced sublimation loss only in the recent past.

(c) $67 \mathrm{P}$ and $103 \mathrm{P} /$ Hartley 2 can be classified in the same category. They appear to have a large probability of staying in orbits with $q<2.5$ au over the last $10000 \mathrm{yr}$. In this event, both of their original sizes could have been substantially larger 


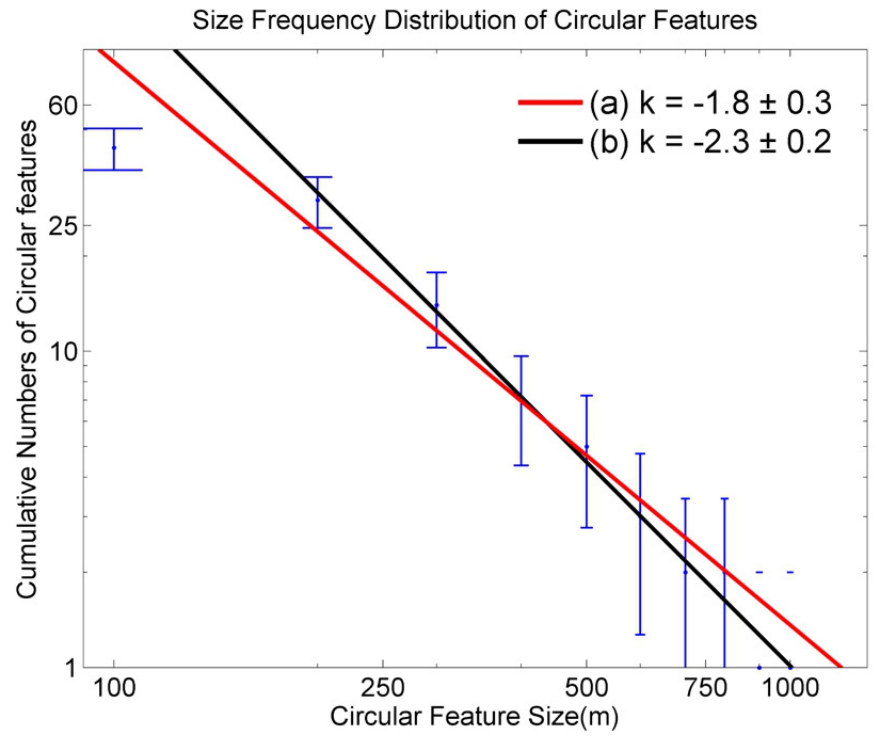

Fig. 8. Cumulative size frequency distribution of the circular depressions on comet $67 \mathrm{P}$. The red line shows the power law distribution computed by including all circular depressions. The blue line is obtained by considering only those with diameter larger than $200 \mathrm{~m}$.

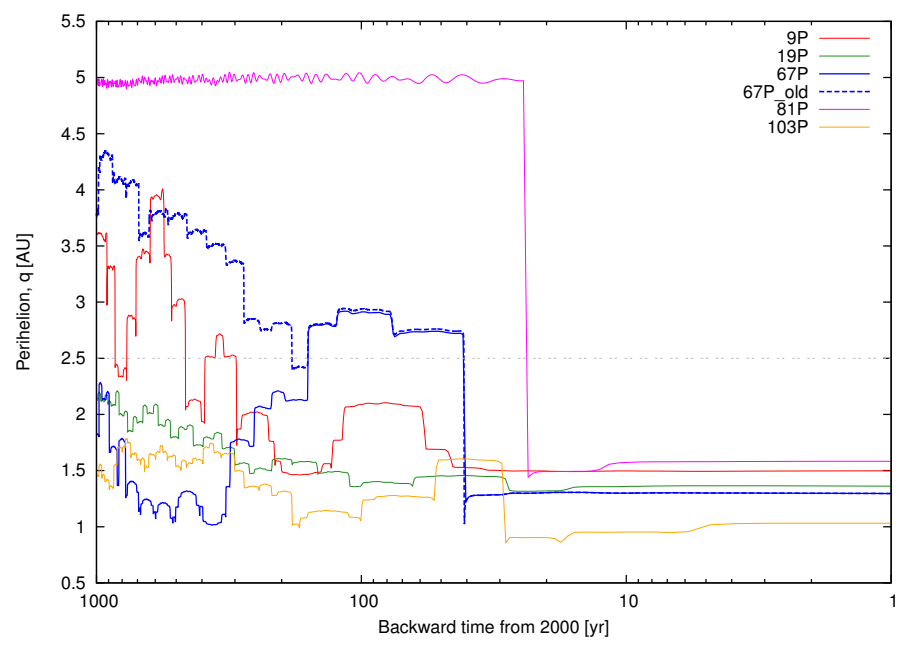

Fig. 9. Comparison of the past orbital evolutions of several short-period comets over one thousand years.

than the present values of $4 \mathrm{~km}$ for 67P (Sierks et al. 2015) and $2.2 \mathrm{~km}$ for 103P (Thomas et al. 2013).

(d) 19P/Borrelly appears to have an even higher probability of having $q<2.5$ au over the integration time interval. The fact that it has a very limited active region $(<10 \%$ of total surface area) according to the imaging results of Soderblom et al. (2002) is probably consistent with the estimate of its old age.

\section{Discussion and summary}

Vincent et al. (2015a), Vincent et al. (2016) showed that the formation of the active pits could probably be related to the sinkhole collapse mechanism possibly followed by thermal stress fracturing the cliff walls surrounding the pits. This means that if local topography allows, continuous cliff fracture and outgassing activity would lead to the expansion of the circumference of the pits. During this process, the residual non-volatile dust and blocks falling on the floor would produce a relatively smooth surface covering the primitive material below. Because of the insulation effect of such a dust mantle, the thermal sublimation rates, either locally or globally, will diminish gradually as the comet repeats its perihelion passages.

While the sizes of the circular depressions would be limited by the surface space available for sublimation, the depths of the steep walls appear to have a maximum of about $200 \mathrm{~m}$. We note that Massironi et al. (2015) analyzed the two-lobe configuration and stratigraphy of comet $67 \mathrm{P}$ and came to the conclusion that these two lobes are two different entities and that each of them has an onion-like structure composed of many layers. The thickness of the stratified layers ( $200 \mathrm{~m}$ at Imhotep) of inhomogeneous compositions and structures might define the maximum depth a circular depression/active pit can reach (see also Pajola et al. 2015). In this simple model, the surface of a JFC after many perihelion passages - could be characterized by relatively smooth topography with the sharp edges eroded away by thermal stress and fracturing as described in Vincent et al. (2016). In this scenario, the north-south dichotomy of the circular depressions distribution on 67P could have its origin in the heterogeneous structure of the comet itself. That is, the evolution of the surface geomorphology might be regulated by the preexisting stratification as discussed in El-Maarry et al. (2015b) and Massironi et al. (2015). Alternatively, the strong asymmetry in solar insolation before and after perihelion (Keller et al. 2015) could be driving the surface and subsurface sublimation processes of different volatilities so that circular depressions could exist preferentially on the northern hemisphere.

Our analysis is complicated by the fact that the solar illumination conditions of the different sides of 67P could have been different in previous orbits (before the last Jupiter encounter, say). That is, even though the northern hemisphere (i.e., the Hapi region) is subject to thicker dust deposition as a consequence of the airfall effect than its sourthern counterpart, the situation could have been reversed in the distant past.

A detailed examination of the circular depressions on the nucleus surface of comet $67 \mathrm{P}$ shows that such steep-wall and flat-bottom structures with sizes between $150 \mathrm{~m}$ and $1 \mathrm{~km}$ have a similar size-frequency distribution to those on comets 9P/Tempel 1 and 81P/Wild 2. The depth-to-diameter ratios of these structures are mostly within the range of $0.1-0.3$. In comparison, the active pits studied by Vincent et al. (2015a) have large $d / D$ ratio $(>0.3)$ and small size $(<300 \mathrm{~m})$. This statistical result coupled with orbital integration studies suggests that the large circular depressions could have outgrown from the small ones via erosive sublimation/mass wasting of the surrounding areas (Britt et al. 2004; Cheng et al. 2013; Vincent et al. 2015a, 2016). However, the exact time scale cannot yet be determined. Either some of these structures are very old and dated back to the early history in the trans-neptunian region, or they could have been generated prior to crossing of the water snow line. In the latter case, sublimation erosion process driven by more volatile ices such as $\mathrm{CO}$ and $\mathrm{CO}_{2}$ could be important. As shown in Kelley et al. (2013), presistent coma activity of a number of JFCs at 3-7 au beyond the water snow line was detected by the Spitzer space telescope. Such outgassing phenomena must have been driven by the sublimation of super-volatile ices.

Finally, it is also understood that statistical descriptions of the orbital histories shown in Fig. 10 are mainly to underline the complex process of the evolution of cometary surface morphology. A case in point is the hyper-active comet, 103P/Hartley 2, which exhibited extreme levels of sublimation in water and $\mathrm{CO}_{2-}$ rich gas jets (A'Hearn et al. 2011). Its volatile behavior does not fit the picture of an old JFC except that it could have followed a low probability and high-perihelion pathway not long ago into 


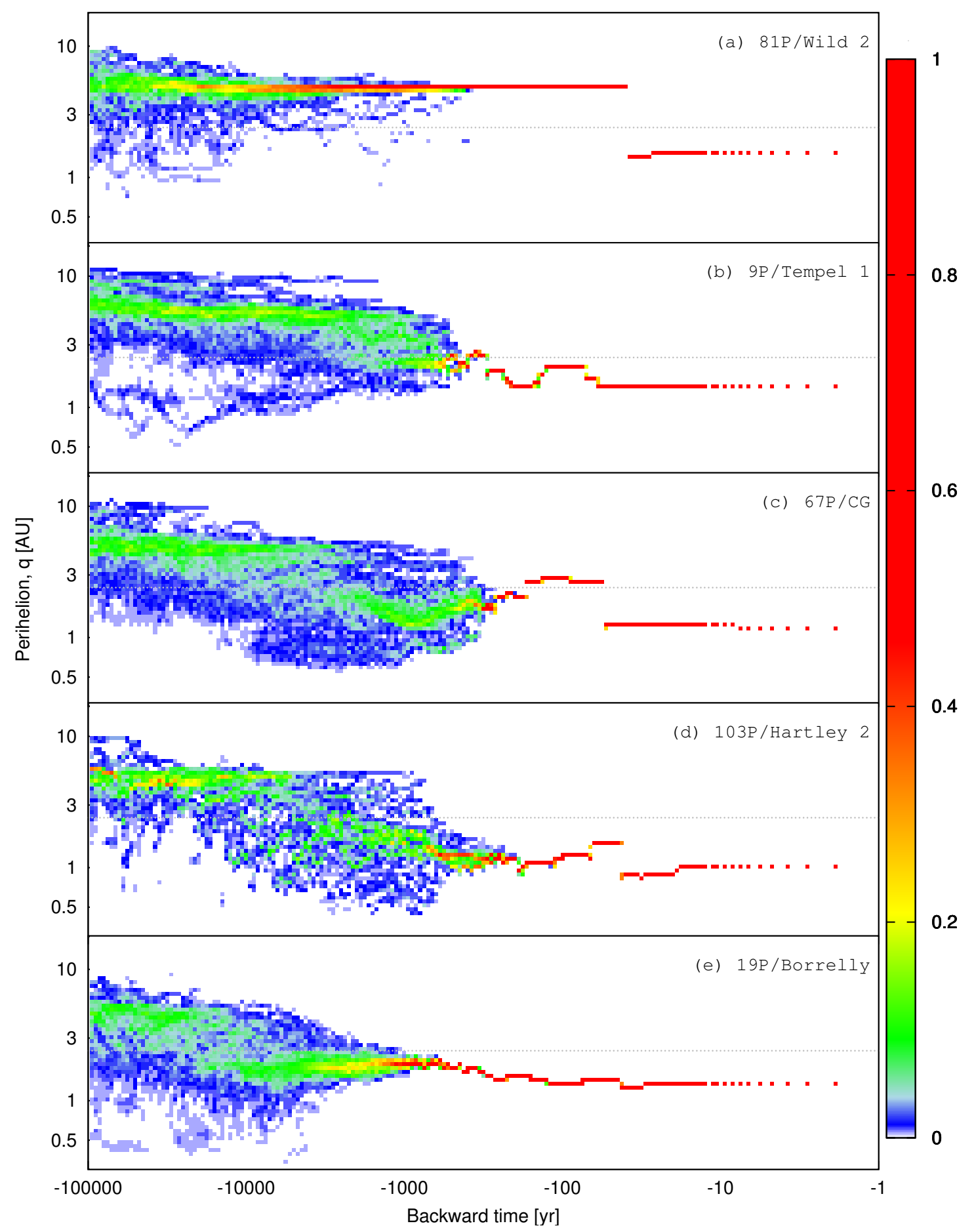

Fig. 10. Comparison of the probable orbital evolutionary histories over the past $100000 \mathrm{yr}$ of five short-period comets visited by spacecraft. The color scale denotes the probability of orbital distribution of a comet in a certain time interval.

the inner solar system. Even so, it is unusual that $103 \mathrm{P} /$ Hartley 2 could remain so active and $\mathrm{CO}_{2}$ rich after crossing the snow line at 2.5 au (A'Hearn et al. 2011; Knight \& Schleicher 2013). This also means that a continuous monitoring of the distant coma activities of comet $67 \mathrm{P} / \mathrm{Churyumov-Gerasimenko} \mathrm{and} \mathrm{other} \mathrm{short-}$ period comets should be pursued in the years to come.

Acknowledgements. OSIRIS was built by a consortium led by the Max-PlanckInstitut für Sonnensystemforschung, Göttingen, Germany, in collaboration with CISAS, University of Padova, Italy, the Laboratoire d'Astrophysique de Marseille, France, the Instituto de Astrofísica de Andalucía, CSIC, Granada, Spain, the Scientific Support Office of the European Space Agency, Noordwijk, Netherlands, the Instituto Nacional de Técnica Aeroespacial, Madrid, Spain, the
Universidad Politéchnica de Madrid, Spain, the Department of Physics and Astronomy of Uppsala University, Sweden, and the Institut für Datentechnik und Kommunikationsnetze der Technischen Universität Braunschweig, Germany. The support of the national funding agencies of Germany (Deutschen Zentrums für Luft- und Raumfahrt), France (Centre National d'Études Spatales), Italy (Agenzia Spaziale Italiana), Spain (Ministerio de Educación, Cultura y Deporte), Sweden (Swedish National Space Board; grant No. 74/10:2), and the ESA Technical Directorate is gratefully acknowledged. We are indebted to the whole Rosetta mission team, Science Ground Segment, and Rosetta Mission Operation Control for their hard work making this mission possible. This work was also supported by grant number MOST 104-2119-M-008-024 (TANGOII), NSC 1022112-M-008-013-MY3, and MOST 104-2111-M-008-020(Space) from the Ministry of Science and Technology of Taiwan, and FDCT grant No. 017/2014/A1 and 039/2013/A2 of Macau Special Administrative Region.

\section{A132, page 8 of 10}




\section{References}

A’Hearn, M. F., Belton, M. J. S., Delamere, W. A., et al. 2011, Science, 332, 1396

Basilevsky, A. T., \& Keller, H. U. 2006, Planet. Space Sci., 54, 808

Belton, M. J. S. 2010, Icarus, 210, 881

Belton, M. J. S., \& Melosh, J. 2009, Icarus, 200, 280

Belton, M. J. S., Thomas, P., Carcich, B., et al. 2013, Icarus, 222, 477

Brasser, R., \& Wang, J.-H. 2015, A\&A, 573, A102

Britt, D. T., Boice, D. C., Buratti, B. J., et al. 2004, Icarus, 167, 45

Brownlee, D. E., Horz, F., Newburn, R. L., et al. 2004, Science, 304, 1764

Bruck Syal, M., Schultz, P. H., Sunshine, J. M., et al. 2013, Icarus, 222, 610

Chambers, J. E. 1999, MNRAS, 304, 793

Cheng, A. F., Lisse, C. M., \& A'Hearn, M. 2013, Icarus, 222, 808

Davidsson, B., Sierks, H., Guettler, C., et al. 2016, A\&A, in press, DOI: 10.1051/0004-6361/201526968

El-Maarry, M. R., Thomas, N., Giacomini, L., et al. 2015a, A\&A, 583, A26

El-Maarry, M. R., Thomas, N., Gracia-Berná, A., et al. 2015b, Geophys. Res. Lett., 42, 5170

Fernández, J. A., Gallardo, T., \& Brunini, A. 2002, Icarus, 159, 358

Fernández, J. A., Sosa, A., Gallardo, T., \& Gutiérrez, J. N. 2014, Icarus, 238, 1

Groussin, O., Hahn, G., Lamy, P. L., Gonczi, R., \& Valsecchi, G. B. 2007, MNRAS, 376, 1399

Groussin, O., Sierks, H., Barbieri, C., et al. 2015, A\&A, 583, A36

Housen, K. R., \& Holsapple, K. A. 2003, Icarus, 163, 102

Keller, H. U., Mottola, S., Davidsson, B., et al. 2015, A\&A, 583, A34

Kelley, M. S., Fernández, Y. R., Licandro, J., et al. 2013, Icarus, 225, 475

Knight, M. M., \& Schleicher, D. G. 2013, Icarus, 222, 691

Lamy, P. L., Toth, I., Fernandez, Y. R., \& Weaver, H. A. 2004, The sizes, shapes, albedos, and colors of cometary nuclei (Tucson: University of Arizona Press), 223

Lara, L. M., Lowry, S., Vincent, J.-B., et al. 2015, A\&A, 583, A9

Levison, H. F., \& Duncan, M. J. 1994, Icarus, 108, 18

Lin, Z.-Y., Ip, W.-H., Lai, I.-L., et al. 2015, A\&A, 583, A11

Lin, Z.-Y., Lai, I.-L., Su, C.-C., et al. 2016, A\&A, 588, L3

Maquet, L. 2015, A\&A, 579, A78

Massironi, M., Simioni, E., Marzari, F., et al. 2015, Nature, 526, 402

Pajola, M., Vincent, J.-B., Güttler, C., et al. 2015, A\&A, 583, A37

Preusker, F., Scholten, F., Matz, K.-D., et al. 2015, A\&A, 583, A33

Rickman, H., Marchi, S., A'Hearn, M. F., et al. 2015, A\&A, 583, A44

Schultz, P. H., Eberhardy, C. A., Ernst, C. M., et al. 2007, Icarus, 190, 295

Sekanina, Z. 2003, J. Geophys. Res., 108, 8112

Sierks, H., Barbieri, C., Lamy, P. L., et al. 2015, Science, 347, 1044

Soderblom, L. A., Becker, T. L., Bennett, G., et al. 2002, Science, 296, 1087

Thomas, P. C., A'Hearn, M. F., Veverka, J., et al. 2013, Icarus, 222, 550

Thomas, N., Sierks, H., Barbieri, C., et al. 2015, Science, 347, 0440

Vincent, J.-B., Bodewits, D., Besse, S., et al. 2015a, Nature, 523, 63

Vincent, J.-B., Oklay, N., Marchi, S., Höfner, S., \& Sierks, H. 2015b, Planet. Space Sci., 107, 53

Vincent, J.-B., Oklay, N., Pajola, M., et al. 2016, A\&A, 587, A14

Yasui, M., Arakawa, M., Hasegawa, S., Fujita, Y., \& Kadono, T. 2012, Icarus, 221,646

1 Institute of Astronomy, National Central University, 32054 ChungLi, Taiwan e-mail: wingip@astro.ncu.edu.tw

2 Institute of Space Sciences, National Central University, 32054 Chung-Li, Taiwan
3 Space Science Institute, Macau University of Science and Technology, Macau

${ }^{4}$ Dept. of Earth Science, National Central University, 32054 ChungLi, Taiwan

5 Max-Planck Institut für Sonnensystemforschung, Justus-vonLiebig-Weg, 3, 37077 Göttingen, Germany

${ }^{6}$ Research and Scientific Support Department, European Space Agency, 2201 Noordwijk, The Netherlands

7 Department of Physics and Astronomy "G. Galilei", University of Padova, Vic. Osservatorio 3, 35122 Padova, Italy

8 Centro di Ateneo di Studi ed Attivitá Spaziali "Giuseppe Colombo", University of Padova, via Venezia 15, 35131 Padova, Italy

9 Aix-Marseille Université, CNRS, LAM (Laboratoire d'Astrophysique de Marseille) UMR 7326, 13388 Marseille, France

10 Centro de Astrobiologia (INTA-CSIC), Madrid, Spain

11 International Space Science Institute, Hallerstrasse 6, 3012 Bern, Switzerland

12 Department of Physics and Astronomy, Uppsala University, Box 516, 75120 Uppsala, Sweden

13 PAS Space Research Center, Bartycka 18A, 00716 Warszawa, Poland

14 Institut für Geophysik und extraterrestrische Physik (IGEP), Technische Universität Braunschweig, 38106 Braunschweig, Germany

15 Department of Astronomy, University of Maryland, College Park, MD 20742-2421, USA

16 LESIA-Observatoire de Paris, CNRS, UPMC Univ. Paris 06, Univ. Paris-Diderot, 5 place J. Janssen, 92195 Meudon Principal Cedex, France

17 LATMOS, CNRS/UVSQ/IPSL, 11 boulevard d'Alembert, 78280 Guyancourt, France

18 INAF Osservatorio Astronomico di Padova, vic. dell Osservatorio 5, 35122 Padova, Italy

19 CNR-IFN UOS Padova LUXOR, via Trasea 7, 35131 Padova, Italy

20 Department of Industrial Engineering, University of Padova, via Venezia 1, 35131 Padova, Italy

21 University of Trento, via Sommarive 9, 38123 Trento, Italy

22 Physikalisches Institut, University of Bern, 3012 Bern, Switzerland

23 INAF - Osservatorio Astronomico di Trieste, via Tiepolo 11, 34143 Trieste, Italy

24 Instituto de Astrofísica de Andalucía (CSIC), c/Glorieta de la Astronomía, 18008 Granada, Spain

25 Deutsches Zentrum für Luft- und Raumfahrt (DLR), Institut für Planetenforschung, Rutherfordstrasse 2, 12489 Berlin, Germany

26 European Space Astronomy Centre (ESAC), PO Box 78, 28691 Villanueva de la Cañada, Madrid, Spain

27 Centre for Astrophysics and Planetary Science, School of Physical Sciences, The University of Kent, Canterbury CT2 7NH, UK

28 Southwest Research Institute, Boulder, CO, USA

29 Department of Information Engineering, University of Padova, via gradenigo 6/B, 35131 Padova, Italy

30 MTA CSFK Konkoly Observatory, 1121 Budapest, Hungary 


\section{Appendix A: Table}

Table A.1. Coordinates of comet 67P's circular depressions.

\begin{tabular}{|c|c|c|c|c|c|c|c|}
\hline ID & Long. & Lat. & Depth (m) & Diameter (m) & $d / D$ & ID in Vincent et al. (2015) & Jet detected \\
\hline A1 & -147.44 & 16.32 & 21 & 192 & 0.11 & Ash-04 & NO \\
\hline $\mathrm{A} 2$ & -152.6 & 13.71 & 17 & 153 & 0.11 & & \\
\hline A3 & -156.09 & 12.44 & 42 & 180 & 0.24 & & \\
\hline A4 & -155.6 & 8.73 & 42 & 126 & 0.34 & Ash-03 & NO \\
\hline A5 & -153.2 & 18.04 & 21 & 182 & 0.12 & & \\
\hline A6 & -150.52 & 25.15 & 12 & 80 & 0.16 & & \\
\hline A7 & -166.13 & 37.63 & 63 & 339 & 0.19 & & \\
\hline A8 & -167.71 & 30.6 & 13 & 116 & 0.12 & & \\
\hline A9 & 140.85 & 33.69 & 95 & 513 & 0.19 & & \\
\hline A 10 & 163.53 & 32.94 & 6 & 170 & 0.03 & & \\
\hline A 11 & 152.28 & 24.48 & 15 & 218 & 0.07 & & \\
\hline A12 & 163.78 & 22.82 & 81 & 265 & 0.31 & & \\
\hline $\mathrm{F} 1$ & -44.59 & -0.73 & 20 & 115 & 0.18 & Ma'at-04 & NO \\
\hline M1 & -5.89 & 4.33 & 129 & 943 & 0.14 & & \\
\hline $\mathrm{M} 2$ & -16.73 & 8.64 & 69 & 167 & 0.42 & & \\
\hline M3 & -19.36 & 2.44 & 43 & 180 & 0.24 & & \\
\hline M4 & -22.43 & 5.11 & 31 & 172 & 0.18 & & \\
\hline M5 & -25.74 & 0.37 & 23 & 213 & 0.11 & & \\
\hline M6 & -0.83 & 45.23 & 65 & 125 & 0.52 & Ma'at-01 & YES \\
\hline M7 & 6.95 & 43.09 & 60 & 130 & 0.47 & Ma'at-02 & YES \\
\hline M8 & 8.53 & 36.9 & 50 & 140 & 0.36 & Ma'at-03 & NO \\
\hline M9 & 19.32 & 33.84 & 26 & 151 & 0.18 & & \\
\hline $\mathrm{P} 1$ & 111.19 & 14.06 & 43 & 265 & 0.17 & & \\
\hline $\mathrm{P} 2$ & 108.8 & -11.93 & 63 & 438 & 0.15 & & \\
\hline P3 & 110.62 & -30.54 & 31 & 465 & 0.07 & & \\
\hline $\mathrm{S} 1$ & -140.88 & 13.58 & 44 & 140 & 0.31 & & \\
\hline $\mathrm{S} 2$ & -143.67 & 16.9 & 64 & 300 & 0.22 & Ash-05 & $\mathrm{NO}$ \\
\hline S3 & -137.81 & 15.39 & 21 & 82 & 0.25 & & \\
\hline $\mathrm{S} 4$ & -131.23 & 15.29 & 87 & 310 & 0.29 & Ash-06 & NO \\
\hline S5 & -123.49 & 7.35 & 9 & 344 & 0.03 & & \\
\hline S6 & -128.12 & 19.77 & 52 & 283 & 0.19 & & \\
\hline $\mathrm{S} 7$ & -118.41 & 1 & 29 & 127 & 0.23 & & \\
\hline S8 & -117.36 & 0.76 & 13 & 110 & 0.12 & & \\
\hline S9 & -113.85 & -5.2 & 56 & 170 & 0.33 & & \\
\hline S10 & -115.77 & 2.79 & 49 & 79 & 0.62 & & \\
\hline S11 & -117.65 & 8.11 & 52 & 193 & 0.27 & & \\
\hline S12 & -119.15 & 14.52 & 38 & 125 & 0.3 & & \\
\hline S13 & -121.01 & 26.21 & 59 & 212 & 0.28 & & \\
\hline S14 & -113.95 & 24.55 & 40 & 261 & 0.16 & & \\
\hline S15 & -124.62 & 45.8 & 210 & 230 & 0.92 & Seth-05 & YES \\
\hline S16 & -142.36 & 63.2 & 50 & 210 & 0.24 & Seth-06 & NO \\
\hline S17 & 119.48 & 83.32 & 234 & 236 & 1 & & \\
\hline S18 & 165.85 & 70.74 & 155 & 677 & 0.23 & & \\
\hline S19 & -149.66 & 53.62 & 185 & 220 & 0.85 & Seth-01 & YES \\
\hline S20 & -134.35 & 39.61 & 44 & 131 & 0.34 & & \\
\hline S21 & -136.27 & 35.49 & 22 & 79 & 0.27 & & \\
\hline $\mathrm{S} 22$ & -143.51 & 35.68 & 34 & 115 & 0.3 & & \\
\hline $\mathrm{S} 23$ & -149.01 & 42.09 & 59 & 200 & 0.3 & & \\
\hline $\mathrm{S} 24$ & -152.8 & 53.82 & 95 & 110 & 0.87 & Seth-02 & YES \\
\hline $\mathrm{S} 25$ & -156.36 & 52.72 & 100 & 140 & 0.72 & Seth-03 & YES \\
\hline S26 & -166.26 & 53 & 39 & 162 & 0.24 & & \\
\hline S27 & -171.63 & 56.73 & 37 & 91 & 0.41 & & \\
\hline S28 & -152.21 & 34.8 & 66 & 213 & 0.31 & & \\
\hline S29 & 83.64 & 57.28 & 65 & 190 & 0.35 & & \\
\hline $\mathrm{S} 30$ & 63.3 & 46.18 & 159 & 272 & 0.59 & & \\
\hline S31 & 58.71 & 25.48 & 89 & 280 & 0.32 & & \\
\hline
\end{tabular}

Notes. S14, S15, S16, S17, S30, S31 are half-pits. 